\title{
WIGGLERS AND SINGLE-PARTICLE DYNAMICS IN THE NLC DAMPING RINGS
}

\author{
M. Venturini* and A. Wolski, LBNL, Berkeley CA 94720, USA \\ A. Dragt, U. of Maryland, College Park MD 20742, USA
}

\begin{abstract}
Wiggler insertions are expected to occupy a significant portion of the lattice of the Next Linear Collider (NLC) Main Damping Rings (MDR) and have a noticeable impact on the single-particle beam dynamics. Starting from a realistic 3D representation of the magnetic fields we calculate the transfer maps for the wigglers, accounting for linear and nonlinear effects, and we study the beam dynamics with particular attention paid to the Dynamic Aperture (DA). A DA reduction is observed but appears to remain within acceptable limits.
\end{abstract}

\section{INTRODUCTION}

The need for a larger momentum compaction in order to tame the effects of collective instabilities has motivated a redesign of the current NLC MDR lattice [1]. The strength of the magnetic field in the dipoles was decreased causing a reduction of radiation loss and therefore requiring longer wiggler insertions to maintain the desired damping. The wiggler insertions now occupy about $61.6 \mathrm{~m}$ of the $300 \mathrm{~m}$ ring circumference (up from $46.2 \mathrm{~m}$ ) adding further linear and nonlinear perturbations to the lattice. An accurate assessment of these effects is desirable in order to obtain a proper tuning of the linear lattice and determine the impact on the Dynamic Aperture (DA). A sufficiently large DA is required for a good injection efficiency (at injection the NLC MDR's should be capable of accommodating a beam with $150 \mathrm{~mm}$-mrad normalized emittance in both transverse planes and a full width $\pm 1 \%$ energy spread).

A previous study [2] showed that the effects of the wiggler nonlinearities in the earlier design of the NLC MDR lattice while noticeable did not introduce unacceptable degradation of the DA. The study made use of a suitable fitting of the magnetic field in the wiggler midplane and a simplified symplectic integrator for tracking the orbits of individual particles. A similar conclusion was reached after a first investigation of the new lattice [1] that was carried out with the wigglers modelled as sequences of standard elements (combined function dipoles, thin octupoles, and drifts). The parameters for these standard elements were tuned in such a way as to reproduce the same horizontal and vertical kicks through a wiggler period as obtained from the more accurate symplectic integrator technique employed earlier.

In this paper we report on a further study on the new lattice using a method that makes some improvements on the field representation and avoids some of the simplifications in the integrator mentioned above. The method requires

\footnotetext{
*mventurini@lbl.gov
}

one component of the magnetic field (for instance obtained with the aid of a magnet design code) on the surface of a cylinder coaxial to and contained within the wiggler aperture. This is sufficient information to determine the field in the interior of the cylindrical surface in the form of a full 3D multipole expansions, properly accounting for the longitudinal dependence of the fields. Upon suitable truncation of this series expansion we determine the corresponding transfer map through third order in the dynamical variables using the code MARYLIE3.0 [4].

\section{WIGGLER FIELD ANALYSIS}

In a current-free region the magnetic field can be expressed in terms of a scalar potential $\psi$, which solves the Laplace equation. For a periodic structure of period $\lambda_{w}$ the most general such scalar potential can be written in cylindrical coordinates as $\psi=\sum_{m=0}^{\infty} \psi_{m}(\rho, z) \sin m \phi$ + term proportional to $\cos m \phi$, where $\psi_{m}(\rho, z)=$ $\sum_{p=-\infty}^{\infty} e^{2 \pi i p z / \lambda_{w}} I_{m}(2 \pi p / \lambda \rho) b_{m, p}, I_{m}$ are the modified Bessel functions, and $b_{m, p}$ arbitrary coefficients. The terms proportional to $\cos m \phi$ correspond to the 'skew' components of the fields, are absent in a error-free planar wiggler and will be neglected here. The azimuthal number $m=1,2,3 \ldots$ corresponds to the dipole, quadrupole, sextupole, ... components (the $m=0$ solenoidal term is also absent in an ideally built wiggler). Alternatively, one can express $\psi_{m}$ as a power series in the radial variable $\rho$ in the form: $\psi_{m},(\rho, z)=\sum_{\ell=0}^{\infty}(-1)^{\ell} \frac{m !}{2^{2 \ell} \ell !(\ell+m) !} C_{m}^{[2 \ell]}(z) \rho^{2 \ell+m}$, where $C_{m}^{[2 \ell]}(z)$, defined by $C_{m}^{[2 \ell]}(z)=$ $\frac{(-1)^{\ell}}{\sqrt{2 \pi}} \frac{1}{2^{m} m !} \sum_{p=-\infty}^{\infty} e^{2 \pi i p z / \lambda_{w}}\left(\frac{2 \pi p}{\lambda_{w}}\right)^{2 \ell+m} b_{m, p}, \quad$ is the $2 \ell$ derivative of $C_{m}^{[0]}(z) \equiv C_{m}(z)$. We will refer to the functions $C_{m}(z)$ as 'generalized gradients'. A field expansion in the current-free region is uniquely determined by specification of the generalized gradients.

Suppose the radial component of the magnetic field is given on the surface of a cylinder of radius $R$ in terms of the Fourier series $B_{\rho}=\sum_{m=0}^{\infty} B_{m}(R, z) \sin (m \phi)$. Then, it can be easily shown that the coefficients $b_{m, p}$ are related to the Fourier integral $\tilde{B}_{m, p}=$ $\frac{1}{\lambda_{w}} \int_{0}^{\lambda_{w}} d z e^{-i 2 \pi p z / \lambda_{w}} B_{m}(R, z)$ of the field data $B_{m}$ by $b_{m, p}=\tilde{B}_{m, p} \lambda_{w} /\left[2 \pi p I_{m}^{\prime}\left(2 \pi p R / \lambda_{w}\right)\right]$. Notice that because of its exponential behavior the Bessel function $I_{m}^{\prime}$ in the denominator of the above expression has a filtering effect on high frequency noise possibly present in the field data [3].

A full 3D field map for a MDR wiggler period is available based on the preliminary design discussed in [5]. The wiggler period is $\lambda_{w}=27 \mathrm{~cm}$, the magnetic field peak 
value $2.1 \mathrm{~T}$. Because the (anti-)symmetry of the fields under a $180^{\circ}$ rotation is enforced exactly only the $2(2 n+1)$ harmonics are present (dipole, sextupole, decapole, ...). Analysis of the radial component of this field at $R=9 \mathrm{~mm}$ results in the profiles of the generalized gradients shown in Fig. 1.
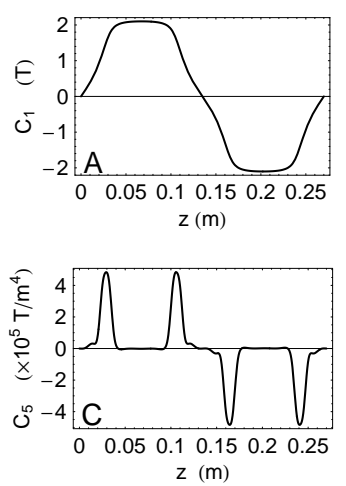
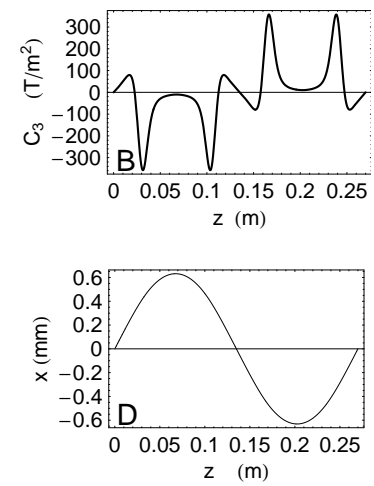

Figure 1: Generalized gradients $C_{m}(z)$ for one period of the MDR wigglers corresponding to the dipole $m=1$ (A), sextupole $m=3$ (B), and decapole $m=5$ (C) field components. The reference orbit is also reported (D).

\section{MAP COMPUTATION}

As an alternative to integrating the equations of motion for the individual particles we use knowledge of the magnetic fields for a wiggler period to determine the corresponding transfer map. We carry out the calculation in a canonical framework using the deviations coordinates from the reference orbit. This requires going through the following steps: determination of the vector potential associated with the field 3D multipole expansion, calculation of the reference orbit, re-expansion of the vector potential (and Hamiltonian) as Taylor series with respect to the deviation coordinates. The Hamiltonian in the latter form is then suitable for calculating the transfer map upon solving the equation of motion - a task carried out by MARYLIE3.0 built-in routines. The resulting map through third order has the Lie representation $\mathcal{M}=\cdots M \exp \left(: f_{3}:\right) \exp \left(: f_{4}:\right)$, where $M$ is the $6 \times 6$ matrix describing the linear part of the dynamics, while the $f_{\ell}$ 's are homogeneous polynomial of degree $\ell$ in the dynamical variables. The matrix $M$ depends only on the quadratic part of the Hamiltonian; $f_{3}$ depends also on the cubic part; $f_{4}$ also on the quartic part. These two generators are connected to quadratic (and higher), cubic (and higher) terms of the map in Taylor form respectively. For straight magnetic elements (in which the reference orbit follows the magnet axis) each individual $2 n$-pole component of the magnetic field contributes only to the $f_{\ell}$ generator with $\ell \geq n$ (the main contribution is absorbed by the generator with $\ell=n$ while the contribution to the generators with $\ell>n$ is related to the $z$-varying part of the generalized gradient - a fringe effect). However, because in wigglers the reference orbit deviates from the $z$-axis, expressing the field in the deviation coordinates (from the magnet frame coordinates) produces a cascade of feed-down terms affecting the generators with $\ell<n$ as well. For example, a sextupole component introduces a correction to the linear part of the dynamics (one can think of this term as a quadrupole-like component); a decapole component affects $f_{4}$ (octupole-like), $f_{3}$, (sextupole-like), etc. These feed-down terms are weighted by increasing powers of the deviation $x_{r}(z)$ of the reference orbit from the wiggler axis. Because for wigglers in high energy machines this amplitude is fairly small $\left(x_{r}(z) \leq 0.6 \mathrm{~mm}\right.$ in our case), in practice for each multipole component only the first feed-down term, which is proportional to $x_{r}(z)$, is significant. For this reason not including higher order multipoles above the decapole in the field representation (as we do) does not affect the calculation of the $3^{\text {rd }}$ order map substantially.

An insight into the relevant terms of the map can be obtained from a simplified analytical calculation under the assumption that the particle orbits (in the deviation variables) do not deviate considerably from straight lines. In such a model the sextupole and decapole components of the field contribute to the dynamics only through feed-down terms. In particular, integrals of the sextupole feed-down term (proportional to $x_{r}(z) C_{3}(z)$ ) contribute to the linear part of the map introducing defocusing horizontally and focusing vertically, while integrals of the decapole feed-down term (proportional to $x_{r}(z) C_{5}(z)$ ) represent the main contribution to the cubic part of the map. No quadratic or quartic terms appear (as would be case for ordinary sextupole or decapole magnets) because the integrals of the $C_{3}(z)$ and $C_{5}(z)$ vanish over a wiggler period. As for the dipole field component, its main effect is to add linear focusing in both transverse planes through the integrals of terms like $x_{r}(z) C_{1}^{[2]}(z)$ - essentially a fringe-effect. The net result on the linear dynamics is significant focusing in the vertical plane (the sextupole feed-down and dipole fringe-field terms add up) and relatively small defocusing in the horizontal plane where the dipole fringe-field and the sextupole feed-down terms have opposite sign (with the latter prevailing slightly). In spite of the approximation involved this simple model appears to be in good quantitative agreement with the MARYLIE numerical calculation for the linear part of the map and reasonable qualitative agreement for the nonlinear part. In particular, the MARYLIE calculation confirms that the dominant nonlinearities are of third order (octupole-like).

In the absence of a detailed design for the ends of the wiggler insertions we used a model consisting of bending magnets and two thin octupole and sextupole lenses.

\section{DA STUDY}

The new MDR lattice is discussed in detail in [1]. For this study we switched off the rf cavities and did not include any errors in the magnets or wigglers. Tracking was 
carried out element by element using the MARYLIE symplectic integrator. In particular, tracking through the wiggler insertions (consisting of 6 full periods in addition to entry and exit ends) was done by propagating the particle orbits through each individual period, using the maps determined from the field data - as discussed in the previous Section. All the other elements where modelled using the MARYLIE standard element library with the inclusion of the MARYLIE built-in modelling of fringe-field effects in the hard-edge limit. In this study the physical aperture of the vacuum chamber ( $8 \mathrm{~mm}$ radius in the wigglers, 20 mm everywhere else) was not accounted for. The DA of the MDR lattice is affected by both the sextupole magnet and wiggler nonlinearities. An assessment of the relative impact of the two sources of nonlinearities is obtained by comparison of the two pictures of Fig. 2. These and the following figures show the DA through 500 turns in the $x-y$ plane located in the middle of the first quadrupole magnet downstream the injection point (the beta-functions are about $\beta_{x}=16 \mathrm{~m}$ and $\beta_{y}=1.6 \mathrm{~m}$ ). The particles are launched at this location with vanishing transverse momenta. The half-ellipse corresponding to $15 \times$ the injected beam size - a plausible DA target - is also shown. Fig. 2A is relative to a lattice in which the wiggler nonlinearities are artificially masked away (sextupoles dominate); Fig. 2B refers to a lattice with the sextupoles turned off (wigglers nonlinearities dominate).
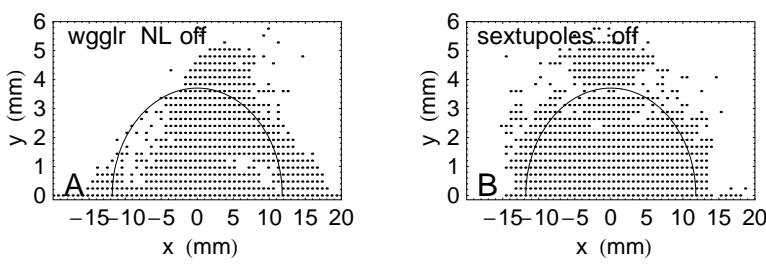

Figure 2: On momentum DA with wiggler nonlinearities turned off (A) and chromatic sextupoles turned-off (B)

The combined effect of chromatic sextupoles and wiggler nonlinearities on the dynamic aperture are shown in Fig 3A for on-momentum particles and Fig.'s 3B and 3C for off-momentum particles $(+1 \%$ and $-1 \%$ respectively). The $15 \sigma$ boundary appears to be mostly respected by onmomentum particles while a somewhat larger degradation of the DA is observed for off-momentum particles.

Finally, Fig. 3D shows the on-momentum DA for a representation of the wiggler periods constructed using combined function dipoles of length $\lambda_{w} / 4$ and thin octupoles. The peak field in the dipoles was set to $2.28 \mathrm{~T}$, so as to give the design integrated squared field strength while the field gradient $(-1.66 \mathrm{~T} / \mathrm{m}$, defocusing horizontally) was adjusted to give a linear transfer map for one wiggler period close to the map calculated by numerical integration through the wiggler field (the individual entries of the linear matrix entries deviate less than 1\%); the strength of the octupole lens was tuned to fit the : $x^{4}:$ Lie generator of the same map. Both maps result in similar horizontal and vertical
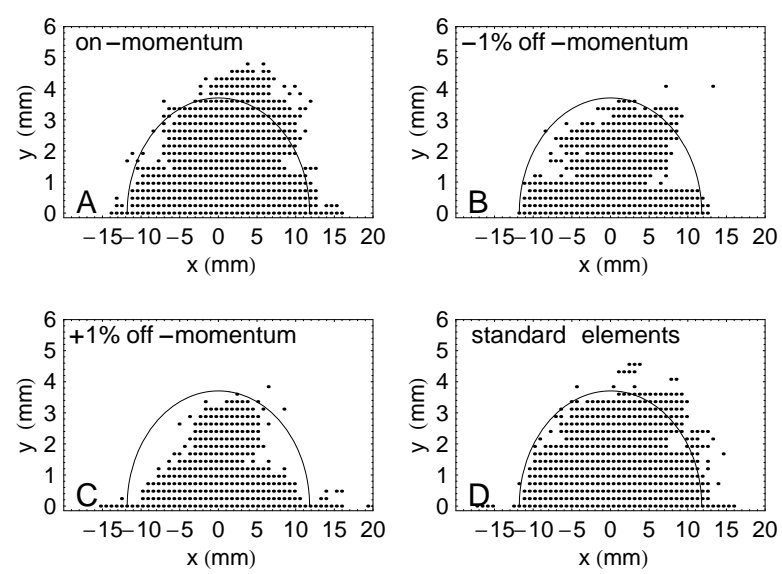

Figure 3: Dynamic aperture for model with wiggler map integrated through fields $(\mathrm{A}, \mathrm{B}, \mathrm{C})$ and model using standard elements (D) - on momentum.
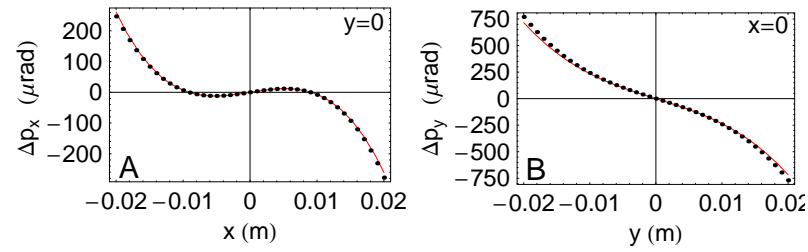

Figure 4: Horizontal (A) and vertical (B) kicks through one wiggler period from map calculated by integration through actual fields (dots) and map constructed from standard elements (line).

kicks through a wiggler period (Fig.4) - with some noticeable discrepancies emerging (not shown) when both $x \neq 0$ and $y \neq 0$ - a reflection of the fact that the nonlinear generators of the two maps (in particular those related to $x-y$ coupling) are not identical. Nevertheless, on-momentum the DA aperture resulting from using the standard element model for the wiggler (Fig. 3D) does not appear very different from that resulting from the more accurate modelling (Fig. 3A). However, a larger discrepancy can be noted in the off-momentum cases (not shown) with the calculation done using the standard-element model giving a somewhat more pessimistic estimate of the DA.

Work supported by the US DOE under Contract DEAC03-76SF00098.

\section{REFERENCES}

[1] M. Woodley and A. Wolski, NLC Tech Note LCC-0113 (2003); A. Wolski et al., (these Proceedings).

[2] A. Wolski et al., PAC01, Proceedings, p. 3798 (2001).

[3] M. Venturini and A. Dragt, NIM-A 415417 (1998).

[4] A. Dragt et al., MARYLiE3.0, Users' Manual, Univ. of Maryland Dept. of Physics Technical Report (May 2003).

[5] J. Corlett et al., CBP Tech. Note 199, LBNL, Berkeley (1999), (also NLC Tech Note LCC-0031). 